Topic: 2. Ischemic Heart Disease

\title{
Differences in Clinical Characteristics, Results and Management of Patients Referred for Pharmacologic Cardiac Stress SPECT Depending on the Protocol Used
}

\author{
Pablo Del Castillo, ${ }^{1}$ Núria Casanovas, ${ }^{1}$ Marcel Santaló, ${ }^{1}$ Fernando Narro, ${ }^{1}$ Ana Paula Caresia, ${ }^{2}$ Ana Barradas, ${ }^{1}$ \\ Walter Bragagnini, ${ }^{1}$ Joan Carles Martin, ${ }^{2}$ Laura Guillamon, ${ }^{1}$ Lluís Bernà, ${ }^{2}$ Antoni Martínez-Rubio ${ }^{1}$ \\ 1. Hospital Universitari Parc Taulí - Cardiology, 2. Hospital Universitari Parc Taulí - Nuclear Medicine
}

Keywords: Radionuclide imaging, Stress, Vasodilator agents, Ischemia, Prognosis

\section{Introduction and Objectives}

Pharmacologic cardiac stress SPECT (PS-SPECT) is recommended for non-invasive evaluation of ischemic heart disease (IHD) in patients unable to exercise. Drugs used for stress in our centre are Dipyridamole (DIP) and Dobutamine (DBT). DIP can be associated to exercise (ex-DIP). The objective of the study is to evaluate the diagnostic capacity of the different strategies.

\section{Materials and Methods}

Retrospective study of 838 patients referred for PS-SPECT between 2010 and 2016.

\section{Results}

DIP was the main agent used for stress (730 tests), followed by DBT (63 tests) and combined ex-DIP (45 tests). DIP is preferred to DBT for its best security profile, reserving DBT to patients with contraindications for DIP administration. Basal characteristics of the patients were similar except for younger age in ex-DIP (69 vs 74 years- old) and lower proportion of women in the DBT group (36,5\% DBT vs $57,5 \%$ DIP). Overall, they had high prevalence of cardiovascular risk factors and chronic IHD. Complications of the test were similar for DIP and DBT $(19,8 \%$ vs $18,6 \%)$ and lower for ex-DIP (10,3\%). Hypotension was the main complication for DIP and arrhythmias and hypotension for DBT. Gammagraphic ischemia was detected in similar percentages with the different protocols (39\% with DIP, $44.4 \%$ with ex-DIP and $44.9 \%$ with DBT). However, number of invasive angiography was lower for DBT group (10.6\% vs $14.6 \%$ DIP and $16.1 \%$ ex-DIP) and revascularization higher for ex-DIP (10.3\%) compared to DIP and DBT groups (7.2\% and $6.7 \%)$. Mortality after a mean follow-up of 20 months, was higher for DBT (24,5\%), followed by DIP (16,6\%) and ex-DIP $(9,4 \%)$.

\section{Conclusions}

PS-SPECT is currently reserved to older and sicker patients. Different protocols of study found similar rates of gammagraphic ischemia. Patients unable to exercise, especially those with contraindications for DIP administration, represent a high-risk profile of patients that are infrequently referred for invasive strategy independently of the result of the study and have high mortality risk. We should rethink about indication of this kind of tests in that kind of patients, tests that can be harmful, expensive and sometimes useless.

\footnotetext{
Comparison of long-term mortality risk following normal exercise vs adenosine myocardial perfusion SPECT. Rozanski et al. Journal of Nuclear Cardiology 2010;17(6):999-1008. Symptom-limited exercise combined with dipyridamole stress: Prognostic value in assessment of known or suspected coronary artery disease by use of gated SPECT imaging. Ahlberg A et al. Journal of Nuclear Cardiology 2008;15(1):42-56.
}

3. Survival by Stress Modality in Patients With a Normal Myocardial Perfusion Study. Johnson N et al. Am J Cardiol 2011;107:986-989. 